\title{
Isolement de bactéries rhizosphériques et effet de leur inoculation en pots chez Zea mays
}

\author{
Jacques FAGES \& Daniel MULARD \\ Pioneer France-Maïs, 4, rue Paul-Berniès, F 31075 Toulouse
}

RÉSUMÉ

\begin{abstract}
Deux souches bactériennes, une fixatrice d'azote appartenant à l'espèce Azospirillum lipoferum et une non fixatrice Enterobacter cloacae ont été isolées de la rhizosphère du maìs en utilisant la méthode du modèle spermosphère. Leur utilisation pour inoculer Zea mays lors d'un essai en pots, a conduit à une forte augmentation de la matière sèche avec Azospirillum et à un effet moindre mais significatif sur certains paramètres avec Enterobacter. Cette approche globale incluant l'isolement et l'inoculation apparaît comme une voie intéressante pour obtenir une promotion de la croissance végétale.
\end{abstract}

Mots clés additionnels : Azospirillum lipoferum, Enterobacter cloacae, fixation de l'azote, Pseudomonas diminuta.

Two bacterial strains, one nitrogen fixer belonging to the specie Azospirillum lipoferum and one non-fixing Enterobacter cloacae, were isolated from the maize rhizosphere using the "spermosphere model" method. When used to inoculate Zea mavs in a pot experiment, A. lipoferum gave a strong beneficial effect on dry matter while $E$. cloacae gave a smaller but significant effect on several parameters. This global approach, including isolation and inoculation, seems to be promising way of promoting plant growth.

Additional key words : Azospirillum lipoferum, Enterobacter cloacae, nitrogen fixation, Pseudomonas diminuta.

\section{INTRODUCTION}

Les plantes de grande culture entretiennent une microflore associée à leurs racines. Ces associations rhizosphériques, en particulier celles mettant en jeu des micro-organismes fixateurs d'azote, ont fait l'objet de nombreuses études lors de la dernière décennie.

Des essais d'inoculation sur diverses graminées sont reportés dans la littérature. Des effets sur le développement racinaire ont été signalés tels que le développement des poils absorbants, le degré de branchement, l'élongation ou le poids des racines (JAIN \& PATRIQUIN, 1984 ; KAPULNIK et al., 1985 ; LINDBERG et al., 1985 ; OKON \& KAPULNIK, 1986). De nombreuses augmentations de poids de matière sèche des parties aériennes ont également été relatées sur le blé (BASHAN, 1986 ; MERTENS \& HESS, 1984 ; REYNDERS \& VLASSAK, 1982), le riz (RINAUDO, 1983), le maïs (LIN et al., 1983 ; O'HARA et al., 1981) et d'autres graminées à grains ou fourragères (MERTENS \& HESS, 1984).
Enfin, le rendement est lui aussi augmenté par l'inoculation (BASHAN, 1986; VLASSAK, 1983) bien que certains résultats négatifs aient été également publiés (ELMERICH, 1984 ; RINAUdO, 1983 ; SMITH et al., 1984).

C'est probablement une conjonction de plusieurs phénomènes qui explique les effets observés (GASKINS et al., 1984 ; OKON, 1985) : fixation biologique de l'azote (BODDEY et al., 1986), production d'hormones de croissance végétale (GASKINS \& HUBBELL, 1979 ; LIN et al., 1983), activité pectinolytique permettant une meilleure absorption minérale (LIN et al., 1983 ; OKON, 1985), compétitivité de l'inoculum (GASKINS et al., 1984)...

Certains auteurs ont montré qu'il existait une certaine spécificité entre les bactéries rhizosphériques et la plante-hôte en mettant en évidence des réponses chimiotactiques (MANDIMBA et al., 1986) et des colonisations préférentielles par certaines espèces (BALDANI \& DÖBEREINER, 1980). Un processus d'isolement de bac- 
téries diazotrophes mettant en jeu l'affinité des bactéries de la rhizosphère pour les exsudats racinaires a reçu le nom de modèle spermosphère (THOMASBAUZON et al., 1982).

Cet article décrit l'isolement de bactéries de la rhizosphère du maïs, selon un processus inspiré de celuici, leur caractérisation et l'utilisation de 2 souches pour l'inoculation en pots de cette même plante.

\section{MATÉRIELS ET MÉTHODES}

\section{A. Processus d'isolement}

30,0 grammes de racines de maïs en fin de cycle végétatif (variété hybride DEA), provenant d'un sol d'alluvions de monoculture de maïs de la plaine de la Garonne, sont lavés puis stérilisés superficiellement par contact d'une minute avec une solution d'éthanol à 70 p. 100, rincés à l'eau distillée stérile, broyés au mortier et dilués dans $300 \mathrm{ml}$ d'eau distillée stérile pour constituer la dilution $10^{-1}$.

Des dilutions décimales de $10^{-2}$ à $10^{-\gamma}$ de ce broyat de racines servent à ensemencer des tubes spermosphères contenant $15 \mathrm{ml}$ de milieu à raison de $0,5 \mathrm{ml}$ par tube et de 3 tubes par dilution. Ces tubes sont préparés selon la méthode de THOMAS-BAUZON et al. (1982) avec une graine de maïs (variété hybride DEA) mise à germer pendant $24 \mathrm{~h}$ et préalablement stérilisée en surface par passages successifs dans : $\mathrm{Ca}(\mathrm{ClO})_{2}$ saturé pendant $2 \mathrm{~h}, \mathrm{H}_{2} \mathrm{O}$ distillée stérile, $\mathrm{H}_{2} \mathrm{O}_{2}$ (11 volumes) pendant $30 \mathrm{~min}, \mathrm{H}_{2} \mathrm{O}$ distillée stérile.

Après croissance pendant $96 \mathrm{~h}$, le contenu des tubes (racines et milieu) présentant un développement bactérien est broyé, dilué dans de l'eau distillée stérile, les différentes dilutions $10^{-5}$ à $10^{-8}$, étant étalées sur boîtes de Petri à raison de $0,1 \mathrm{ml}$ par boîte et de 3 boîtes par dilution. Ces boîtes contiennent le milieu de WATANABE \& BARRAQUIO (1979) modifié selon THOMAS-BAUZON et al. (1982).

Toutes les colonies isolées et dont la culture a été entretenue sur le milieu ci-dessus sont purifiées et caractérisées par l'étude des caractères culturaux, l"utilisation des galeries d'identification bactérienne API 20E et API 50CH (API System, 38390 La balme les grottes, France) ainsi que divers tests biochimiques classiquement utilisés en microbiologie tels la recherche des enzymes respiratoires et de la respiration anaérobie des nitrates, l'étude des métabolismes glucidique et protidique, la coloration de Gram.

Les souches sont également testées pour leur capacité à fixer l'azote atmosphérique selon le test de réduction de l'acétylène. Une mesure qualitative est effectuée par mise sous 1 p. 100 d'acétylène d'un tube contenant le milieu de Watanabe modifié fraîchement ensemencé. L'éthylène est dosé par chromatographie en phase gazeuse au bout de $24 \mathrm{~h}$ (chromatographe Intersmat IGC $112 \mathrm{FL}$, détecteur à ionisation de flamme, gaz vecteur $\mathrm{N}_{2}$ ). Une mesure quantitative pour les souches diazotrophes est ensuite effectuée en modèle spermosphère, les tubes étant mis sous 10 p. 100 d'acétylène $48 \mathrm{~h}$ après ensemencement. L'éthylène est dosé $6 \mathrm{~h}$ après l'ajout d'acétylène puis toutes les $24 \mathrm{~h}$ pendant $5 \mathrm{j}$.

Cette mesure sera exprimée par plante (en nmoles $\mathrm{C}_{2} \mathrm{H}_{4} \mathrm{~h}^{-1}$ plante ${ }^{-1}$ ) car le développement racinaire de la plantule conditionne le nombre de bactéries coloni- satrices et donc la quantité d'acétylène réduit et ce quelle que soit la taille de l'inoculum.

\section{B. Inoculation en pots}

Les 2 souches bactériennes utilisées pour cet essai sont: Azospirillum lipoferum crtl et Enterobacter cloacae crt3, issues toutes 2 du processus d'isolement décrit ci-dessus.

Le support utilisé est un mélange $50 / 50(\mathrm{v} / \mathrm{v})$ de sable d'Antibes et de vermiculite (Vermex M ; Elfi). Le sable est préalablement rincé dans plusieurs bains d'eau déminéralisée et tamisé à $1 \mathrm{~mm}$. Pour obtenir une bonne homogénéité le mélange est réalisé en présence d'1 1 d'eau pour 51 de mélange dans un mélangeur rotatif de $200 \mathrm{l}$.

Le support après homogénéisation est stérilisé par autoclave à $121{ }^{\circ} \mathrm{C}$ pendant $30 \mathrm{mn}$ en bocaux de $2 \mathrm{l}$.

9 graines de maïs (variété hybride DEA) stérilisées par passage dans $\mathrm{Ca}(\mathrm{ClO})_{2}$ pendant $2 \mathrm{~h}$ et $\mathrm{H}_{2} \mathrm{O}_{2}$ (11 volumes) pendant $30 \mathrm{mn}$, rincées plusieurs fois dans l'eau distillée stérile, sont déposées à la surface du support dans chaque pot. Une épaisseur d'environ $1 \mathrm{~cm}$ de support recouvre ensuite les graines.

L'inoculation est réalisée, à raison de $10^{9}$ bactéries par graine, avec une suspension cellulaire préalablement centrifugée et resuspendue dans le milieu nutritif de compensation d'évapotranspiration décrit cidessous et contenant pour les 2 souches $3,310^{9}$ bactéries/ml. Le dénombrement des bactéries est effectué par la méthode NPP (nombre le plus probable).

Les souches crt 1 et crt 3 ont été cultivées 24 h sur un agitateur orbital à $30^{\circ} \mathrm{C}$. Les milieux utilisés ont les compositions suivantes. Pour Azospirillum : $\mathrm{NH}_{4} \mathrm{Cl}$ $2,5 \mathrm{~g}, \mathrm{CaCl}_{2} 20 \mathrm{mg}, \mathrm{NaCl} 100 \mathrm{mg}, \mathrm{MgSO}_{4} 7 \mathrm{H}_{2} \mathrm{O}$ $200 \mathrm{mg}, \mathrm{FeSO}_{4} 7 \mathrm{H}_{2} \mathrm{O} 10 \mathrm{mg}, \mathrm{Na}_{2} \mathrm{MoO}_{4} 2 \mathrm{H}_{2} \mathrm{O} 2 \mathrm{mg}$, $\mathrm{K}_{2} \mathrm{HPO}_{4} 6 \mathrm{~g}, \mathrm{KH}_{2} \mathrm{PO}_{4} 4 \mathrm{~g}$, glucose $10 \mathrm{~g}$, extrait de levure $3 \mathrm{~g}, \mathrm{H}_{2} \mathrm{O}$ q.s.p. 11. Pour Enterobacter: $\mathrm{KH}_{2} \mathrm{PO}_{4} 3 \mathrm{~g}, \mathrm{~K}_{2} \mathrm{HPO}_{4} 2 \mathrm{~g}$, peptone $3 \mathrm{~g}$, glucose $10 \mathrm{~g}$, $\mathrm{H}_{2} \mathrm{O}$ q.s.p. 11. Les témoins sont constitués d'une suspension cellulaire de la souche autoclavée.

Pour compenser l'évapotranspiration on utilise un milieu nutritif dont la composition est la suivante: $\mathrm{KNO}_{3} 384 \mathrm{mg}, \mathrm{K}_{2} \mathrm{HPO}_{4} 52 \mathrm{mg}, \mathrm{KH}_{2} \mathrm{PO}_{4} 109 \mathrm{mg}$, $\mathrm{NaNO}_{3} 17 \mathrm{mg}, \mathrm{NaCl} 12 \mathrm{mg}, \mathrm{NH}_{4} \mathrm{NO}_{3} 160 \mathrm{mg}$, $\mathrm{Ca}\left(\mathrm{NO}_{3}\right)_{2} 4 \mathrm{H}_{2} \mathrm{O} 732 \mathrm{mg}, \mathrm{MgSO}_{4} 7 \mathrm{H}_{2} \mathrm{O} 185 \mathrm{mg}$, $\mathrm{FeSO}_{4} 7 \mathrm{H}_{2} \mathrm{O} 1 \mathrm{mg}, \mathrm{ZnSO}_{4} 7 \mathrm{H}_{2} \mathrm{O} 1 \mathrm{mg}, \mathrm{H}_{3} \mathrm{BO}_{3} 1 \mathrm{mg}$,

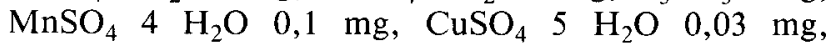
$\left(\mathrm{NH}_{4}\right)_{2} \mathrm{MoO}_{4} 0,03 \mathrm{mg}, \mathrm{H}_{2} \mathrm{O}$ q.s.q. 11 .

Tous les milieux sont stérilisés par autoclavage à $121{ }^{\circ} \mathrm{C}$ pendant $30 \mathrm{mn}$.

La méthode des blocs a été utilisée avec un nombre de répétitions égal à 12 . Les plantes ont été cultivées à $20^{\circ} \mathrm{C}$ avec une durée d'éclairement quotidienne de $15 \mathrm{~h}$. La récolte a été réalisée au bout de $42 \mathrm{j}$. Les paramètres élongation et évapotranspiration ont été mesurés 3 fois par semaine. L'évapotranspiration était mesurée par pesée et compensée après chaque mesure. Les autres mesures, poids de matière sèche des parties aériennes et racinaires, teneur en azote et en phosphore des feuilles, ont été effectuées à la récolte.

L'analyse des résultats a été effectuée à l'aide d'une analyse de variance informatisée (test de Fisher) ; un calcul de p.p.d.s. (plus petite différence significative) est effectué pour comparer les traitements 2 à 2 (test de Student) lorsque le test de Fisher révèle un effet. 


\section{RÉSULTATS}

\section{A. Processus d'isolement}

C'est à partir des modèles spermosphères ayant reçu les dilutions $10^{-2} 10^{-4}$ et $10^{-7}$ que nous avons effectué les isolements et purifications sur milieu de Watanabe modifié. Nous avons choisi de nous limiter aux tubes présentant la croissance bactérienne autour des racines la plus importante, et ce, pour une dilution faible, une dilution intermédiaire et une dilution élevée.

Les souches bactériennes, toutes gram négatives, qui ont ainsi pu être isolées, étaient au nombre de 13.

La caractérisation des types culturaux, du type respiratoire, des enzymes de la respiration, des métabolismes glucidique et protidique a permis de déterminer le genre dans la plupart des cas (10 sur 13) et l'espèce pour un plus petit nombre d'entre eux (6 sur 13). Le tableau 1 résume ces résultats.

Deux souches appartenant aux espèces Azospirillum lipoferum et Pseudomonas diminuta sont capables de réduire l'acétylène en éthylène.

Le développement bactérien, le long des racines est nettement perceptible de visu. Tout au long des $5 \mathrm{j}$ de mesure, la production d'éthylène en fonction du temps a été linéaire. Les résultats sont consignés dans le tableau 2. On observe une activité nitrogénasique beaucoup plus importante dans le cas de l'Azospirillum. L'essai d'inoculation sera effectué avec les souches Azospirillum lipoferum crtl et Enterobacter cloacae crt3, cette dernière étant la souche dominante de notre isolement.

\section{B. Inoculation en pots}

Comme le montrent les figures 1,2 et 3 un effet positif dû à l'inoculation est observé sur les poids de matière sèche racinaire et foliaire, l'élongation et l'évapotranspiration. Pour ces variables l'ordre crt1-crt3témoin est toujours respecté. Seules les différences de poids de matière sèche racinaire ne sont pas statistiquement significatives au seuil de 5 p. 100 .

On enregistre également une teneur en phosphore significativement plus élevée dans les plantes inoculées avec la souche crt3 par rapport aux plantes témoins. La souche crt3 confère également une teneur en eau supérieure aux parties aériennes. Un effet analogue a déjà été cité pour Azospirillum (OKON, 1985).

Le tableau 3 résume les principaux résultats.

Nous avons contrôlé la colonisation des racines par les souches inoculées : à partir de racines provenant de pots inoculés nous avons pu re-isoler des souches présentant la même morphologie et le même profil biochimique que les souches crt 1 et $\operatorname{crt} 3$.

TABLEAU 1

Identification des 13 souches bactériennes issues du processus d'isolement. Identification of the 13 bacterial strains from the isolation procedure.

\begin{tabular}{cllll}
\hline Numéro & \multicolumn{1}{c}{ Genre } & \multicolumn{1}{c}{ Espèce } & Fixateur d'azote & Appellation \\
\hline 1 & Enterobacter & cloacae & - & crt3 \\
2 & Pseudomonas & cepacia & - & cr12 \\
3 & Pseudomonas & diminuta & - & \\
4 & Pseudomonas & pseudoalcaligenes & - & \\
5 & Pseudomonas & stutzeri & - & \\
6 & Pseudomonas & n.d. & + & \\
7 & Azospirillum & lipoferum & - & \\
8 & Acinetobacter & n.d. & - & \\
9 & Achromobacter & n.d. & - & \\
10 & Alcaligenes & n.d. & - & \\
11 & n.d. & & - & \\
12 & n.d. & & \\
13 & n.d. &
\end{tabular}

n.d. : non déterminé.

TABLEAU 2

Activité réductrice d'acétylène (ARA) : mesure quantitative en modèle spermosphère. Acetylene reduction activity (ARA) : quantitative measurement in spermosphere model.

\begin{tabular}{|c|c|c|}
\hline Développement racinaire & $\begin{array}{c}\text { ARA* }^{*} \text {. lipoferum } \\
\text { (nmoles } \mathrm{C}_{2} \mathrm{H}_{4} \cdot \mathrm{h}^{-1} \cdot \text { plante }^{-1} \text { ) }\end{array}$ & $\begin{array}{c}\text { ARA* }^{*} P \text {. diminuta } \\
\text { (nmoles } \mathrm{C}_{2} \mathrm{H}_{4} \cdot \mathrm{h}^{-1} \text {. plante }{ }^{-1} \text { ) }\end{array}$ \\
\hline$+t$ & 58 & 28 \\
\hline+ & 38 & $\begin{array}{r}20 \\
9\end{array}$ \\
\hline
\end{tabular}

$+\quad$ : faible développement racinaire.

++ : fort développement racinaire.

: moyenne de 4 répétitions $(\sigma=3,64)$. 


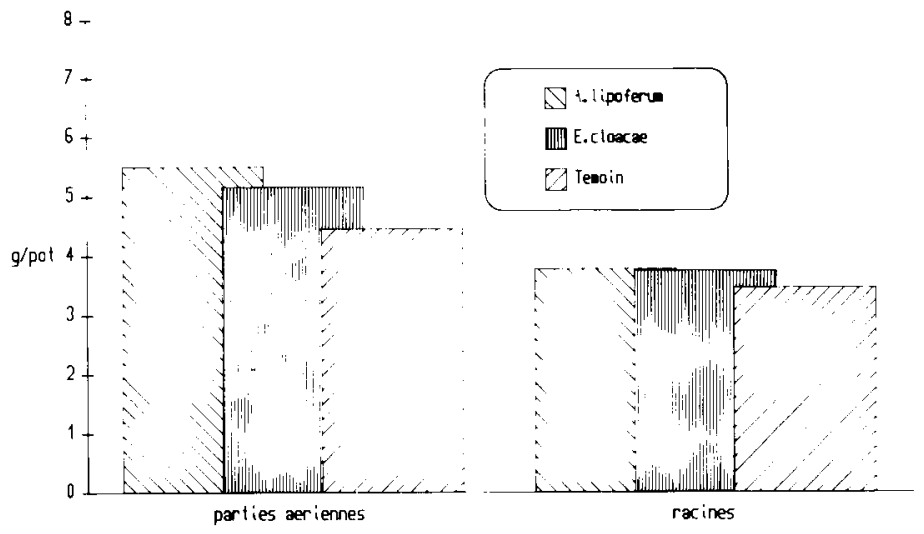

Figure 1

Effets de l'inoculation sur le poids de matière sèche des racines et des parties aériennes. Inoculation effects on root and shoot dry weights.

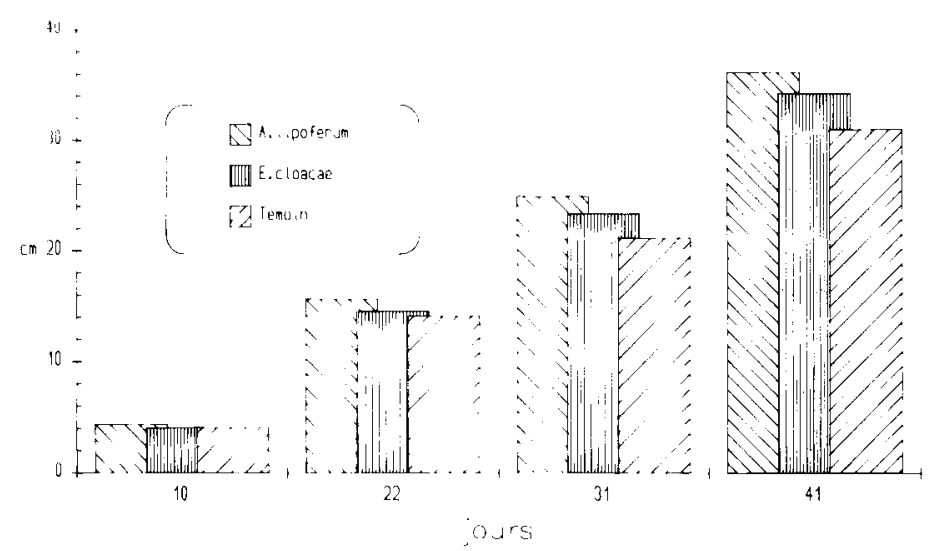

Figure 2

Effets de l'inoculation sur l'élongation des plantules.

Inoculation effects on shoot length.

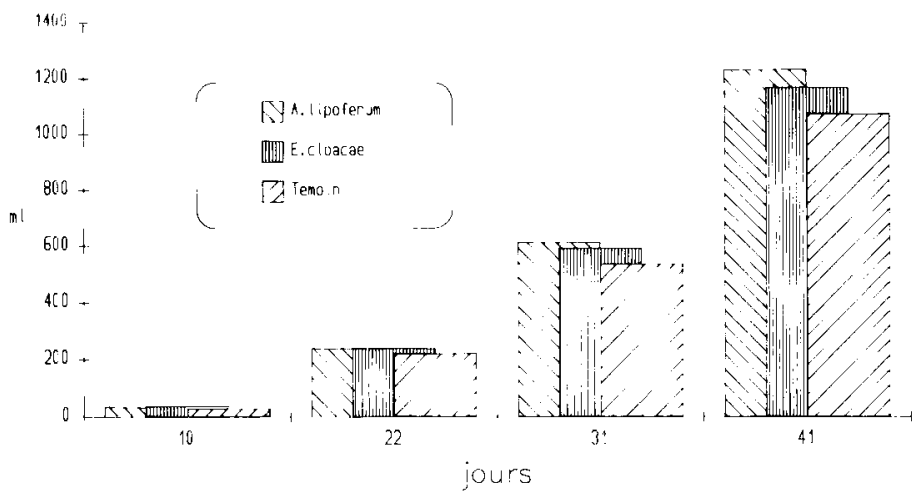

Figure 3

Effets de l'inoculation sur l'évapotranspiration.

Inoculation effects on evapotranspiration. 
TABLEAU 3

Effets de l'inoculation de Zea mays par A. lipoferum crtl et E. cloacae crt3.

Effects of A. lipoferum crtl and $\mathrm{E}$. cloacae crt3 inoculation on Zea mays.

\begin{tabular}{|c|c|c|c|c|}
\hline & & Témoin & crt 1 & $\operatorname{crt} 3$ \\
\hline Poids M.S. racinaire & (g/pot) & $3,47^{\mathrm{a}}$ & $3,79^{\mathrm{a}}$ & $3,76^{\mathrm{a}}$ \\
\hline Poids M.S. foliaire & (g./pot) & $4,46^{\mathrm{a}}$ & $5,51^{\mathrm{b}}$ & $5,17^{\mathrm{h}}$ \\
\hline Elongation & $(\mathrm{cm})$ & $31,1^{\text {a }}$ & $36,2 b$ & 34,3 ab \\
\hline Evapotranspiration & $\left(\mathrm{cm}^{3} / \mathrm{pot}\right)$ & $1073^{a}$ & 1233 b & 1168 ab \\
\hline Teneur en eau & $(\%)$ & $9,77^{\mathrm{a}}$ & $10,15^{\mathrm{ab}}$ & $10,42^{\mathrm{b}}$ \\
\hline Teneur en azote & $(\%)$ & $3,15^{\mathrm{a}}$ & $3,10^{\mathrm{a}}$ & $3,18^{\mathrm{a}}$ \\
\hline Teneur en phosphore & $(\%)$ & $4,31^{\mathrm{a}}$ & $4,37^{\mathrm{ab}}$ & $4,52^{\mathrm{b}}$ \\
\hline
\end{tabular}

M.S. : matière sèche.

Les valeurs suivies d'une même lettre ne sont pas significativement différentes au seuil de $5 \%$ (test de Student).

\section{DISCUSSION}

\section{A. Processus d'isolement}

S'il n'est pas surprenant de rencontrer un Azospirillum fixateur d'azote dans la rhizosphère du maïs, il est en revanche plus inhabituel de trouver comme espèce diazotrophe un Pseudomonas diminuta, bien que d'autres espèces de ce genre soient connues pour être diazotrophes.

Deux des souches isolées présentent une activité réductrice d'acétylène. Bien que les milieux utilisés tant en modèle spermosphère qu'en boîtes de Petri ne contiennent pas d'azote combiné, le fait que la plante puisse libérer dans ses exsudats des composés azotés (acides aminés...) et que le milieu de Watanabe modifié contienne de l'extrait de levure explique que la microflore sélectionnée contienne à la fois des souches diazotrophes et des souches non fixatrices d'azote.

Une croissance cryptique est à exclure ainsi qu'une association entre fixateurs et non-fixateurs d'azote moléculaire, la purification des souches ayant été établie avec certitude sur plusieurs milieux.

Comme toute méthode d'isolement, celle-ci possède des limitations intrinsèques. En particulier elle ne prétend pas être une " photographie » de la rhizosphère à un moment particulier, la présence du végétal n'étant pas une garantie de fidélité aux processus naturels.

Toutefois elle présente l'avantage d'exercer une pression de sélection en faveur des bactéries présentant une affinité pour les exsudats racinaires de la plante et conduit au couple souche bactérienne-plante hôte le plus efficace selon le critère de la fixation de l'azote : ce critère étant à nos yeux non pas une garantie de promotion de croissance végétale (comme de nombreux auteurs l'ont clairement démontré : GASKINS et al., 1984 ; O'HARA et al., 1981 ; OKON, 1985 ; VLASSAK, 1983) mais plutôt un gage d'analogie avec les conditions réelles de carence en azote auxquelles les microorganismes rhizosphériques sont confrontés du fait de l'absorption des racines. De ce fait la pression de sélection exercée va bien dans le sens d'une affinité entre les bactéries et la plante-hôte.

\section{B. Inoculation en pots}

Bien que les souches inoculées n'aient pas été marquées, il est hautement probable que ce sont bien celles que l'on a retrouvées à la récolte.

L'inoculation avec Azospirillum est fortement bénéfique à la croissance de la plante. L'absence de différences significatives sur la teneur en azote s'explique par le fait que le milieu nutritif n'était pas carencé en azote, la fixation biologique de cette molécule n'a donc probablement pas joué un rôle important dans ce processus.

L'inoculation par Enterobacter cloacae conduit en outre à 2 effets déjà décrits dans la littérature pour d'autres espèces : une augmentation de la teneur en eau et en phosphore des feuilles. Une meilleure absorption des phosphates a été parfois reliée à une activité solubilisatrice de la bactérie (GASKINS et al., 1984). Dans cet essai les phosphates ont été introduits sous forme soluble ce qui élimine cette hypothèse.

Il faut noter que les différents effets observés l'ont été sur des plantes recevant par ailleurs une nutrition minérale optimale ; ceci laisse entrevoir la possibilité d'une synergie au champ entre fertilisation minérale et inoculation bactérienne déjà évoquée par quelques auteurs (REYNDERS \& VLASSAK, 1982).

Cet essai doit être appréhendé comme une première démarche complète reliant intimement les phases d'isolement et d'inoculation. Ces résultats, quoique partiels, sont extrêmement encourageants dans la perspective d'une utilisation au champ de bactéries en conditions d'agriculture intensive. La suite de ce travail consistera en une élucidation des paramètres volontairement omis lors de ce premier essai comme la densité de l'inoculum et en une optimisation du processus d'isolement puis au passage en conditions agricoles au champ.

Reçu le 24 juin 1987. Accepté le 21 février 1988.

\section{REMERCIEMENTS}

Nous remercions Monsieur J. Balandreau du centre de pédologie biologique du C.N.R.S. à Vandœuvre-lès-Nancy (France) pour ses suggestions et ses précieux commentaires sur le manuscrit. 


\section{RÉFÉRENCES BIBLIOGRAPHIQUES}

Baldani V. L., Döbereiner J., 1980. Host plant specificity in the infection of cereals with Azospirillum spp. Soil Biol. Biochem., 12, 434-444.

Bashan Y., 1986. Enhancement of wheat root colonization and plant development by Azospirillum brasilense Cd following temporary depression of rhizosphere microflora. Appl. environ. Microbiol., 51, 1067-1071.

Boddey R. M., Baldani V. L., Baldani J. I., Döbereiner J., 1986. Effect of inoculation of Azospirillum spp. on nitrogen accumulation by field grown wheat. Plant Soil, 95, 109-121.

Elmerich C., 1984. Molecular biology and ecology of diazotrophs associated with non-leguminous plants. Bio-technology, 11, 967-978.

Gaskins M. H., Hubbell D. H., 1979. Response of non-leguminous plants to root inoculation with free-living diazotrophic bacteria. In : J. C. Harley \& R. S. Russell. The soil root interface. Academic Press, N. Y., 448 p.

Gaskins M. H., Albrecht S. L., Hubbell D. H., 1984. Rhizosphere bacteria and their use to increase plant productivity: a review. Agric. Ecos. Environ., 12, 99-116.

Jain D. K., Patriquin D. G., 1984. Root hair deformation, bacterial attachment, and plant growth in wheat-Azospirillum associations. Appl. environ. Microbiol., 48, 1208-1213.

Kapulnik Y., Okon Y., Henis Y., 1985. Changes in root morphology of wheat caused by Azospirillum inoculation. Can. J. Microbiol., 31, 881-887.

Lin W., Okon Y., Hardy R., 1983. Enhanced mineral uptake by Zea mays and Sorghum bicolor roots inoculated with Azospirillum brasilense. Appl. environ. Microbiol., 45, 1775-1779.

Lindberg T., Granhall U., Tomenius K., 1985. Infectivity and acetylene reduction of diazotrophic rhizosphere bacteria in wheat (Triticum aestivum) seedlings under gnotobiotic conditions. Biol. Fertil. Soils, 1, 123-129.
Mandimba G., Heulin T., Bally R., Guckert A., Balandreau J., 1986. Chemotaxis of free-living nitrogen-fixing bacteria towards maize mucilage. Plant Soil, 90, 129-139.

Mertens T., Hess D., 1984. Yield increase in spring wheat (Triticum aestivum) inoculated with Azospirillum lipoferum under greenhouse and field conditions of temperate region. Plant Soil, 82, 87-89.

O'Hara G. W., Davey M. T., Lucas J. A., 1981. Effect of inoculation of Zea mays with Azospirillum brasilense strain under temperate conditions. Can. J. Microbiol., 27, 871-877.

Okon Y., 1985. Azospirillum as a potential inoculant for agriculture. Trends Biotechnol., 9, 223-228.

Okon Y., Kapulnik Y., 1986. Development and function of Azospirillum-inoculated roots. Plant Soil, 90, 3-16.

Reynders L., Vlassak K., 1982. Use of Azospirillum brasilense as biofertilizer in intensive wheat cropping. Plant Soil, 66, 217-223.

Rinaudo G., 1983. Fixation hétérotrophe de l'azote dans la rhizosphère du riz. Thèse de doctorat d'état. Orstom, Paris.

Smith R. L., Schank S. C., Milam J. R., Baltensperger A. A., 1984. Responses of Sorghum and Pennisetum species to the $\mathrm{N}_{2}$-fixing bacterium Azospirillum brasilense. Appl. environ. Microbiol., 47, 13311336.

Thomas-Bauzon D., Weinhard P., Villecourt P., Balandreau J., 1982. The spermosphere model. I. Its use in growing, counting, and isolating $\mathrm{N}_{2}$-fixing bacteria from the rhizosphere of rice. Can. J. Microbiol., 28, 922-928.

Vlassak K., 1983. Réaction du froment d'hiver à des inoculations bactériennes. Rev. agric. Fr., 36, 605-610.

Watanabe I., Barraquio W. L., 1979. Low levels of fixed nitrogen required for isolation of free-living nitrogen fixing organisms from rice roots. Nature, $277,565-566$. 